\title{
$\begin{array}{ll}\text { Research Square } & \begin{array}{l}\text { Preprints are preliminary reports that have not undergone peer review. } \\ \text { They should not be considered conclusive, used to inform clinical practice, } \\ \text { or referenced by the media as validated information. }\end{array}\end{array}$
}

\section{Multi-drug Resistant (MDR), Extended Spectrum Beta-lactamase (ESBL) and New Delhi Metallo Beta-lactamase (NDM) Producing Escherichia Coli in Rescued Sloth Bears (Melursus Ursinus), India}

O.R. Vinodhkumar ( $\nabla$ vinodhkumar.rajendran@gmail.com )

Indian Veterinary Research Institute https://orcid.org/0000-0002-7232-4122

M. Karikalan

IVRI: Indian Veterinary Research Institute

S. Ilayaraja

SOS

Arun A Sha

WILDlife SOS

B.R. Singh

IVRI: Indian Veterinary Research Institute

D.K. Sinha

IVRI: Indian Veterinary Research Institute

S. Chandra Mohan

IVRI: Indian Veterinary Research Institute

A.M. Pawde

IVRI: Indian Veterinary Research Institute

AK Sharma

IVRI: Indian Veterinary Research Institute

\section{Short Report}

Keywords: Sloth bear, carbapenem, ESBL, E. coli, virulence

Posted Date: February 19th, 2021

DOI: https://doi.org/10.21203/rs.3.rs-213170/v1

License: (9) (7) This work is licensed under a Creative Commons Attribution 4.0 International License. Read Full License 


\section{Abstract}

The study reports the MDR, ESBL, and NDM producing Escherichia coli (CRE) isolated from the rescued sloth bear (Melursus ursinus), India. The faecal samples of adult rescued sloth bear $(n=21)$ were collected from a rescue center located in India during 2015-2016 and processed for isolation and antibacterial susceptibility pattern of $E$. coli. $45 E$. coli isolates were recovered, and on phenotypic screening, 23 were MDR, 17 were ESBL producers, and five were carbapenem-resistant (CR). The MDR isolates carried beta-lactamase, chloramphenicol, aminoglycosides, tetracycline, fluroquinone, and sulphadimidine resistance genes. All the phenotypic ESBL producing isolates had blaCTX-M genes. On genotypic screening, three CRE $(60.0 \%, 3 / 5)$ were positive for blaNDM carbapenemase gene. Efflux pump-mediated carbapenem resistance was noticed in two CRE isolates (40.0 \%, 2/5). The CRE also isolates co-harbored AMR genes like blaTEM-1, blaAmpC, qnrA, qnrB, qnrS, tetA, tetB and sull. Virulence screening of the resistant isolates revealed the presence of Stx1, Stx2, eae, hlyA genes.Plasmid incompatibility (Inc) typesof three NDM positive isolates revealed that two isolates blaNDM-5 gene on Incl1 an one isolate on IncF plasmid. Apart from NDM genes, the plasmids also carried tetracycline, beta-lactamase and quinolone resistance genes. The plasmid multilocus sequence typing (pMLST) of the E. coli Incl1 plasmid showed the Sequence Type (ST) 297. This appears to be the first report of multi-drug resistant, extended spectrum betalactamase (ESBL) producing and blaNDM-5geneson Incl1 and IncF plasmids in rescued sloth bear.

\section{Introduction}

Antimicrobial resistance is an emerging global threat and needs immediate attention. Extended spectrum beta-lactamases (ESBL) and/or carbapenemases producing Escherichia coli are of great concern worldwide and is a complex and multidimensional problem involving humans, animals, and the environment (ATTERBY et al. 2017). Carbapenems are one of the most important groups of antimicrobials and are considered as a last line of drugs for the treatment of severe infections. Carbapenem resistance in animals appear to have developed and disseminated because of clinical carbapenem use in human medicine and presence of carbapenem resistance in food animals have been reported (Pruthvishree et al. 2017; Nirupama et al. 2018). Genes conferring AMR can spread quickly, being passed on to bacteria that had never been exposed to the initial selective pressure and thereby disseminating throughout microbial communities (Allen et al. 2010). Wild birds and mammals acts as important reservoirs and potential vectors for the spread of resistant bacteria and genetic determinants of AMR (Caroll et al. 2015). Wild animals are not naturally exposed to antimicrobials; though, they may be infected with antibiotic-resistant bacteria via the acquisition of resistant bacteria from human sources, agricultural facilities, and associated contaminated environments (Dolejska et al. 2015). Antimicrobial agents, such as antibiotics, are used extensively in human and veterinary medicine and often as part of routine agricultural production (Kummerer 2009). The prevalence level of resistant bacteria is linked with human activity (Bonnedahl et al. 2009; Alroy and Ellis 2011). Hence, the study was carried out to identify the molecular epidemiology of multi-drug resistant, extended spectrum beta-lactamase (ESBL) producing and carbapenem resistant Escherichia coli isolated from rescued sloth bear.

\section{Materials And Methods}

A cross-sectional study was conducted in a bear rescue centre for screening of antimicrobial resistance in rescued sloth bears of India during 2015-16. The representative faecal samples from apparently healthy adult sloth bears $(n=21)$ were collected in sterile transport swabs (HiCulture, HiMedia, India). Following enrichment in MacConkey broth at $37^{\circ} \mathrm{C}$ for $6-10 \mathrm{~h}$, the faecal swabs were streaked on MacConkey agar and incubated at $37^{\circ} \mathrm{C}$ for $12-18 \mathrm{~h}$. Two to three lactose-fermenting colonies were streaked on eosin methylene blue (EMB) agar medium and incubated at $37^{\circ} \mathrm{C}$ overnight for preliminary characterization. Then, the colonies showing characteristic metallic sheen on EMB agar were picked up for biochemical tests. The reference strains were retrieved from the Division of Epidemiology, ICAR-Indian Veterinary Research Institute, Izatnagar repository, and used in this study (GenBank accession number: KU318701, KU318691, KU382501).

The biochemically confirmed. E.coli $(\mathrm{n}=45)$ isolates were tested for antibiotic susceptibility pattern with amoxicillin $(10 \mu \mathrm{g})$, amoxicillin/clavulanic acid $(20 / 10 \mu \mathrm{g})$, aztreonam $(30 \mu \mathrm{g})$, chloramphenicol $(30 \mu \mathrm{g})$, ceftriaxone $(30 \mu \mathrm{g})$, cefazolin $(30 \mu \mathrm{g})$, cefpodoxime $(10 \mu \mathrm{g})$, ceftazidime $(30 \mu \mathrm{g}), \mathrm{cef}$.taxime $(30 \mu \mathrm{g})$, cefepime $(30 \mu \mathrm{g})$, cefixime $(5 \mu \mathrm{g})$, cefoxitin $(30 \mu \mathrm{g})$, piperacillin - tazobactam $(100 / 10 \mu \mathrm{g})$, cefotaxime/clavulanic acid $(30 / 10 \mu \mathrm{g})$, tetracycline $(30 \mu \mathrm{g})$, nitrofurantoin $(300 \mu \mathrm{g})$, gentamicin $(10 \mu \mathrm{g})$,kanamycin $(30 \mu \mathrm{g})$, co-trimoxazole $(25 \mu \mathrm{g})$, ciprofloxacin $(5 \mu \mathrm{g})$ and norfloxacin $(10 \mu \mathrm{g})$ by disc diffusion method. For phenotypic identification of ESBL producers, the combination disc method by cefotaxime, ceftazidime with and without clavulanic acid was used (Andrews 2003). The isolates were further confirmed by ESBL E-test (HiMedia, Mumbai, India). Screening for carbapenem resistance was carried out with meropenem (MEM, $10 \mu \mathrm{g})$, imipenem (IMP,10 $\mu \mathrm{g})$, and ertapenem (ETP,10 $\mu \mathrm{g})$ disks (BD, Sparks, MD, USA) along with metal ion chelator ethylene diamine tetra acetic acid (EDTA; $1900 \mathrm{mg}$ ) for metallo beta-lactamase (MBL) production (Pruthvishree et al. 2017). The keyhole reaction between carbapenem drug and EDTA was considered positive for MBL. The MIC for meropenem was determined using E-test strip (EZY MRP, HiMedia, Mumbai, India). The CLSI, 2014 (CLSI 2014) breakpoints were used for interpretation of susceptibility patterns.

A conservative approach was utilized, and only isolates classified as resistant were further analysed for AMR encoding genes. Total DNA was isolated from $E$. coli isolates using a QIAamp ${ }^{\circledR}$ DNA Mini Kit (QIAGEN, Hilden, Germany). The isolates were screened for the presence of antimicrobial resistance genes (ARG), including beta-lactamase, major carbapenemase, sulphonamide, plasmid-mediated quinolone (PMQR), and tetracycline resistance genes. The isolates were also screened for putative virulence genes (Paton and Paton 1998). The reactions were optimized individually in $25 \mu \mathrm{L}$ volumes, with 10 picomole of each individual primer (supplementary table 1). The PCR amplicon from all reactions was electrophoresed in $0.5 \times$ TBE buffer using $1.5 \%$ agarose gel and visualized under UV illumination (Syngene, USA). Representative PCR amplicons were purified and sequenced by commercial sequencing services (Eurofins, Bengaluru, India). The sequencing results were obtained, and homology searches were performed using the BLAST algorithm (http:// http://blast.ncbi.nlm.nih.gov/Blast.cgi). The sequence data were submitted to GenBank, and accession numbers were obtained.

Plasmid DNA was isolated using a QIA prep Spin miniprep kit (QIAGEN, Helsinki, Finland) from the blaNDM positive E. coli. PCR-based replicon typing (PBRT) was used to determine the plasmid incompatibility groups (Johnson et al. 2007). Plasmid MLST was performed using specific primers

Page 2/8 
(https://pubmlst.org/plasmid/primers/) and sequenced by the Sanger method (Eurofins India Ltd, Bengaluru). The sequences were edited using BioEdit v7.0.5 and queried in the plasmid MLST website (https://pubmlst.org/bigsdb?db=pubmlst_plasmid_seqdef) for an allelic profile, sequence type, and clonal complex. The isolates not showing carbapenemase mediated resistance were screened for the presence of active efflux pump by the ethidium bromide cartwheel method.

\section{Statistical analysis}

Antibiotic susceptibility phenotypic profiles and gene presence was converted into binary code. For each antibiotic 0 denoted susceptibility and 1 denoted represented resistance similarly the presence of a gene was designated as 1 and absence as 0 . Principal component analysis (PCA) was performed using the package Factoextra from the open-source statistical program $\mathrm{R}$.

\section{Results And Discussion}

\section{A total of $45 \mathrm{E}$. coli isolates were isolated from 21 sloth bear faecal}

samples and the antibiogram showed that the isolates were resistant to gentamicin $(62.2 \%, 28 / 45)$, cefotaxime $(60.0 \%, 27 / 45)$, ceftazidime $(60.0 \%, 27 / 45)$, cefotaxime/clavulanic acid $(57.8 \%, 26 / 45)$, ceftriaxone (55.6\%, 25/45), ceftazidime /clavulanic acid (55.6\%, 25/45), norfloxacin (55.6\%, 25/45), cefpodoxime $(53.3 \%, 24 / 45)$, cefoxitin $(53.3 \%, 24 / 45)$, piperacillin/tazobactam $(53.3 \%, 24 / 45)$, cefexime $(51.1 \%, 23 / 45)$, ciprofloxacin (51.1\%, $23 / 45)$, tetracycline $(51.1 \%$, 23/45), sulphadimidine/trimethoprim combination (46.7\%, 21/45), cefepime (46.7\%, 21/45), chloramphenicol (42.2\%, 19/45), nitrofurantoin (40.0\%, 18/45), aztreonam $(35.6 \%, 16 / 45)$, meropenem $(11.1 \%, 5 / 45)$, imipenem $(8.9 \%, 4 / 45)$ and ertapenem $(8.9 \%, 4 / 45)$. Of the 45 isolates, $51.1 \%(23 / 45)$ were resistant to more than two classes of screened antibiotics and classified as MDR. The ESBL screening by combined disc method revealed $37.8 \%$ (17/45) isolates as ESBL producers (Table 1). 


\section{S. Isolates Antibacterial resistance pattern}

no

\begin{tabular}{|c|c|c|c|c|c|c|c|c|c|c|c|c|c|c|c|c|c|c|c|c|}
\hline & & AMX & AMC & $\mathrm{CZ}$ & ATZ & СОT & $c x$ & CTX & CFM & CPD & CIP & CAZ & CTR & $\mathrm{K}$ & C & GEN & TE & FOX & $\mathrm{F} / \mathrm{M}$ & TZP \\
\hline 1 & SB-1 & $S$ & $\mathrm{R}$ & $S$ & $S$ & $S$ & $S$ & $S$ & $S$ & $S$ & $S$ & $S$ & $S$ & $S$ & $S$ & S & $S$ & $S$ & S & $S$ \\
\hline 2 & $\begin{array}{l}\text { SB-2 } \\
\text { ESBL }\end{array}$ & $\mathrm{R}$ & $\mathrm{R}$ & $\mathrm{R}$ & $\mathrm{R}$ & $\mathrm{R}$ & $\mathrm{R}$ & $\mathrm{R}$ & $\mathrm{R}$ & $\mathrm{R}$ & $\mathrm{R}$ & $\mathrm{R}$ & $\mathrm{R}$ & $\mathrm{R}$ & $\mathrm{R}$ & $\mathrm{R}$ & $\mathrm{R}$ & $\mathrm{R}$ & $\mathrm{R}$ & $\mathrm{R}$ \\
\hline 3 & $\begin{array}{l}\text { SB-3 } \\
\text { ESBL }\end{array}$ & $\mathrm{R}$ & $\mathrm{R}$ & $\mathrm{R}$ & $S$ & $\mathrm{R}$ & $\mathrm{R}$ & $\mathrm{R}$ & $\mathrm{R}$ & $\mathrm{R}$ & $\mathrm{R}$ & $\mathrm{R}$ & $\mathrm{R}$ & $\mathrm{R}$ & $S$ & $\mathrm{R}$ & $\mathrm{R}$ & $\mathrm{R}$ & $S$ & $\mathrm{R}$ \\
\hline 4 & SB-4 & $\mathrm{R}$ & $S$ & $\mathrm{R}$ & $S$ & $\mathrm{R}$ & $\mathrm{R}$ & $S$ & $S$ & $\mathrm{R}$ & $S$ & $S$ & $S$ & $\mathrm{R}$ & $S$ & S & $S$ & $S$ & S & $S$ \\
\hline 5 & SB-5 & $\mathrm{R}$ & $S$ & $\mathrm{R}$ & $S$ & $S$ & $\mathrm{R}$ & $S$ & $S$ & $S$ & $S$ & $S$ & $S$ & $\mathrm{R}$ & $\mathrm{R}$ & $\mathrm{R}$ & $S$ & $S$ & $S$ & $S$ \\
\hline 6 & SB-6 & $\mathrm{R}$ & $\mathrm{R}$ & $\mathrm{R}$ & $S$ & $\mathrm{R}$ & $\mathrm{R}$ & $S$ & $S$ & $S$ & $S$ & $S$ & $S$ & $\mathrm{R}$ & $S$ & S & $S$ & $\mathrm{R}$ & S & $S$ \\
\hline 7 & SB-7 & $\mathrm{R}$ & $\mathrm{R}$ & $\mathrm{R}$ & $S$ & $\mathrm{R}$ & $\mathrm{R}$ & $\mathrm{R}$ & $\mathrm{R}$ & $\mathrm{R}$ & $\mathrm{R}$ & $\mathrm{R}$ & $\mathrm{R}$ & $\mathrm{R}$ & $\mathrm{R}$ & $\mathrm{R}$ & $\mathrm{R}$ & $\mathrm{R}$ & $\mathrm{R}$ & $\mathrm{R}$ \\
\hline 8 & $\begin{array}{l}\text { SB- } \\
8 \mathrm{ESBL}\end{array}$ & $\mathrm{R}$ & $\mathrm{R}$ & $\mathrm{R}$ & $\mathrm{R}$ & $\mathrm{R}$ & $\mathrm{R}$ & $\mathrm{R}$ & $\mathrm{R}$ & $\mathrm{R}$ & $S$ & $\mathrm{R}$ & $\mathrm{R}$ & $S$ & $\mathrm{R}$ & $\mathrm{R}$ & $\mathrm{R}$ & $\mathrm{R}$ & S & $\mathrm{R}$ \\
\hline 9 & SB-9 & $\mathrm{R}$ & $S$ & $S$ & $S$ & $\mathrm{R}$ & $\mathrm{R}$ & $S$ & $\mathrm{R}$ & $S$ & $\mathrm{R}$ & $S$ & $S$ & $\mathrm{R}$ & $\mathrm{R}$ & S & $S$ & $S$ & S & $\mathrm{R}$ \\
\hline 10 & SB-10 & $\mathrm{R}$ & $S$ & $S$ & $S$ & $\mathrm{R}$ & $S$ & $\mathrm{R}$ & $S$ & $S$ & $\mathrm{R}$ & $S$ & $S$ & $\mathrm{R}$ & $\mathrm{R}$ & $\mathrm{R}$ & $\mathrm{R}$ & $S$ & $S$ & $S$ \\
\hline 11 & SB-11 & $\mathrm{R}$ & $S$ & S & $S$ & $S$ & $S$ & $\mathrm{R}$ & $S$ & $S$ & $\mathrm{R}$ & $S$ & $S$ & $\mathrm{R}$ & $\mathrm{R}$ & S & $S$ & S & S & $S$ \\
\hline 12 & $\begin{array}{l}\text { SB-12 } \\
\text { ESBL }\end{array}$ & $\mathrm{R}$ & $\mathrm{R}$ & $\mathrm{R}$ & $\mathrm{R}$ & $\mathrm{R}$ & $\mathrm{R}$ & $\mathrm{R}$ & $\mathrm{R}$ & $\mathrm{R}$ & $\mathrm{R}$ & $\mathrm{R}$ & $\mathrm{R}$ & $\mathrm{R}$ & $S$ & $\mathrm{R}$ & $\mathrm{R}$ & $\mathrm{R}$ & $\mathrm{R}$ & $\mathrm{R}$ \\
\hline 13 & SB-13 & $\mathrm{R}$ & $\mathrm{R}$ & $\mathrm{R}$ & $\mathrm{R}$ & $\mathrm{R}$ & $\mathrm{R}$ & $\mathrm{R}$ & $\mathrm{R}$ & $\mathrm{R}$ & $\mathrm{R}$ & $\mathrm{R}$ & $\mathrm{R}$ & $\mathrm{R}$ & $\mathrm{R}$ & $\mathrm{R}$ & $\mathrm{R}$ & $\mathrm{R}$ & $\mathrm{R}$ & $\mathrm{R}$ \\
\hline
\end{tabular}

ESBL

$\begin{array}{llllllllllllllllllllllllllllll}14 & \text { SB-14 } & R & S & R & S & S & S & S & S & S & S & S & S & S & R & S & S & S & R & S & R\end{array}$

$\begin{array}{lllllllllllllllllllllllllllll} & \mathrm{SB}- & \mathrm{R} & \mathrm{R} & \mathrm{R} & \mathrm{R} & \mathrm{S} & \mathrm{R} & \mathrm{R} & \mathrm{R} & \mathrm{R} & \mathrm{R} & \mathrm{R} & \mathrm{R} & \mathrm{R} & \mathrm{R} & \mathrm{R} & \mathrm{R} & \mathrm{R} & \mathrm{R} & \mathrm{R} & \mathrm{R}\end{array}$

15ESBL

$\begin{array}{llllllllllllllllllllllllllllll} & \mathrm{SB}-16 & \mathrm{R} & \mathrm{R} & \mathrm{R} & \mathrm{R} & \mathrm{S} & \mathrm{R} & \mathrm{R} & \mathrm{R} & \mathrm{R} & \mathrm{R} & \mathrm{R} & \mathrm{R} & \mathrm{R} & \mathrm{S} & \mathrm{R} & \mathrm{R} & \mathrm{R} & \mathrm{R} & \mathrm{R} & \mathrm{R}\end{array}$ ESBL

$\begin{array}{lllllllllllllllllllllllllll}17 & \text { SB-17 } & \mathrm{R} & \mathrm{R} & \mathrm{R} & \mathrm{R} & \mathrm{R} & \mathrm{R} & \mathrm{R} & \mathrm{R} & \mathrm{R} & \mathrm{R} & \mathrm{R} & \mathrm{R} & \mathrm{R} & \mathrm{S} & \mathrm{R} & \mathrm{R} & \mathrm{R} & \mathrm{R} & \mathrm{R} & \mathrm{R}\end{array}$ ESBL

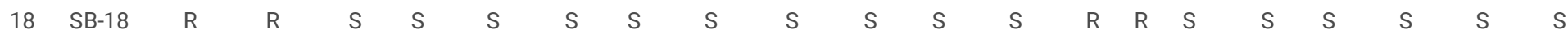

$\begin{array}{lllllllllllllllllllllllll} & \text { SB- } & R & R & R & R & S & R & R & R & R & R & R & R & S & R & R & R & R & R & R & R\end{array}$

$\begin{array}{lllllllllllllllllllllllll}20 & \text { SB }-20 & R & R & R & R & R & R & R & R & R & R & R & R & R & S & R & S & R & S & R & R\end{array}$ ESBL

$\begin{array}{lllllllllllllllllllllllllll}21 & \text { SB-22 } & \text { R } & R & R & S & R & R & R & R & R & R & R & R & R & S & R & R & R & S & R & S\end{array}$ ESBL

$\begin{array}{lllllllllllllllllllllllllllll}22 & \mathrm{SB}-23 & \mathrm{R} & \mathrm{R} & \mathrm{R} & \mathrm{S} & \mathrm{R} & \mathrm{R} & \mathrm{R} & \mathrm{R} & \mathrm{R} & \mathrm{S} & \mathrm{R} & \mathrm{R} & \mathrm{S} & \mathrm{R} & \mathrm{R} & \mathrm{R} & \mathrm{R} & \mathrm{R} & \mathrm{R} & \mathrm{R}\end{array}$ ESBL

$\begin{array}{lllllllllllllllllllllllllllll}23 & \text { SB-24 } & R & S & S & S & S & S & S & S & S & S & R & S & R & S & S & S & S & S & S & S\end{array}$

$\begin{array}{llllllllllllllllllllllllllllll}24 & \text { SB-25 } & \text { S } & R & S & S & S & S & R & S & R & R & S & S & R & S & R & R & R & S & S & S\end{array}$

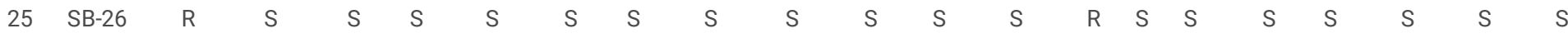

$\begin{array}{llllllllllllllllllllllllllllll}26 & \mathrm{SB} 27 / & \mathrm{R} & \mathrm{R} & \mathrm{R} & \mathrm{R} & \mathrm{R} & \mathrm{R} & \mathrm{R} & \mathrm{R} & \mathrm{R} & \mathrm{R} & \mathrm{R} & \mathrm{R} & \mathrm{R} & \mathrm{R} & \mathrm{R} & \mathrm{R} & \mathrm{R} & \mathrm{R} & \mathrm{R} & \mathrm{R}\end{array}$

$\begin{array}{llllllllllllllllllllllllllll} & \mathrm{SB} 28 / & \mathrm{R} & \mathrm{R} & \mathrm{S} & \mathrm{R} & \mathrm{R} & \mathrm{R} & \mathrm{R} & \mathrm{R} & \mathrm{R} & \mathrm{R} & \mathrm{R} & \mathrm{R} & \mathrm{R} & \mathrm{R} & \mathrm{R} & \mathrm{R} & \mathrm{R} & \mathrm{R} & \mathrm{R} & \mathrm{R}\end{array}$

$\begin{array}{llllllllllllllllllllllllllllllllllll}28 & \mathrm{SB} 28 / & \mathrm{R} & \mathrm{R} & \mathrm{R} & \mathrm{R} & \mathrm{R} & \mathrm{R} & \mathrm{R} & \mathrm{R} & \mathrm{R} & \mathrm{R} & \mathrm{R} & \mathrm{R} & \mathrm{S} & \mathrm{R} & \mathrm{R} & \mathrm{R} & \mathrm{R} & \mathrm{R} & \mathrm{R} & \mathrm{R}\end{array}$ $16-\mathrm{CRE}$

R: resistant ; S: sensitive; AMX: amoxicillin; AMC: amoxicillin-clavulanic acid; ATZ: aztreonam; C: chloramphenicol; CTR: ceftriaxone; CPD: cefpodoxime; CAZ co-trimoxazole; ERP: ertapenem; F/M: nitrofurantoin; FOX: cefoxitin; GEN : gentamicin; IMP: imipenem; K-kanamycin, NX: norfloxacin; MRP- meropenem; TE: $t$ 


\begin{tabular}{|c|c|c|c|c|c|c|c|c|c|c|c|c|c|c|c|c|c|c|c|c|c|}
\hline \multirow{2}{*}{$\begin{array}{l}\text { S. } \\
\text { no }\end{array}$} & \multirow[t]{2}{*}{ Isolates } & \multicolumn{20}{|c|}{ Antibacterial resistance pattern } \\
\hline & & AMX & AMC & $\mathrm{CZ}$ & ATZ & сот & $c X$ & CTX & CFM & CPD & CIP & CAZ & CTR & $K$ & C & GEN & TE & FOX & $F / M$ & TZP & N) \\
\hline 29 & $\begin{array}{l}\text { SB- } \\
29 / 18- \\
\text { CRE }\end{array}$ & $\mathrm{R}$ & $\mathrm{R}$ & $\mathrm{R}$ & $S$ & $S$ & $\mathrm{R}$ & $\mathrm{R}$ & $\mathrm{R}$ & $\mathrm{R}$ & $\mathrm{R}$ & $\mathrm{R}$ & $\mathrm{R}$ & $\mathrm{R}$ & $S$ & $\mathrm{R}$ & $\mathrm{R}$ & $\mathrm{R}$ & $\mathrm{R}$ & $\mathrm{R}$ & $\mathrm{R}$ \\
\hline 30 & $\begin{array}{l}\text { SB-30 } \\
\text { CRE }\end{array}$ & $\mathrm{R}$ & $\mathrm{R}$ & $S$ & $\mathrm{R}$ & $\mathrm{R}$ & $\mathrm{R}$ & $\mathrm{R}$ & $\mathrm{R}$ & $\mathrm{R}$ & $\mathrm{R}$ & $\mathrm{R}$ & $\mathrm{R}$ & $\mathrm{R}$ & $\mathrm{R}$ & $\mathrm{R}$ & $\mathrm{R}$ & $\mathrm{R}$ & $\mathrm{R}$ & $\mathrm{R}$ & $\mathrm{R}$ \\
\hline 31 & $\begin{array}{l}\text { SB-31 } \\
\text { ESBL }\end{array}$ & $\mathrm{R}$ & $\mathrm{R}$ & $\mathrm{R}$ & $\mathrm{R}$ & $\mathrm{R}$ & $\mathrm{R}$ & $\mathrm{R}$ & $\mathrm{R}$ & $\mathrm{R}$ & $\mathrm{R}$ & $\mathrm{R}$ & $\mathrm{R}$ & $\mathrm{R}$ & $S$ & $\mathrm{R}$ & $\mathrm{R}$ & $\mathrm{R}$ & $\mathrm{R}$ & $\mathrm{R}$ & $\mathrm{R}$ \\
\hline 32 & $\begin{array}{l}\text { SB-32 } \\
\text { ESBL }\end{array}$ & $\mathrm{R}$ & $\mathrm{R}$ & $\mathrm{R}$ & $\mathrm{R}$ & $\mathrm{R}$ & $\mathrm{R}$ & $\mathrm{R}$ & $\mathrm{R}$ & $\mathrm{R}$ & $\mathrm{R}$ & $\mathrm{R}$ & $\mathrm{R}$ & $\mathrm{R}$ & $\mathrm{R}$ & $\mathrm{R}$ & $\mathrm{R}$ & $\mathrm{R}$ & $\mathrm{R}$ & $\mathrm{R}$ & $\mathrm{R}$ \\
\hline 33 & $\begin{array}{l}\text { SB-33 } \\
\text { ESBL }\end{array}$ & $\mathrm{R}$ & $\mathrm{R}$ & $\mathrm{R}$ & $S$ & $S$ & $\mathrm{R}$ & $\mathrm{R}$ & $\mathrm{R}$ & $\mathrm{R}$ & S & $\mathrm{R}$ & $\mathrm{R}$ & $\mathrm{R}$ & $S$ & $\mathrm{R}$ & $\mathrm{R}$ & $\mathrm{R}$ & $S$ & $\mathrm{R}$ & $\mathrm{R}$ \\
\hline 34 & $\begin{array}{l}\text { SB-34 } \\
\text { ESBL }\end{array}$ & $\mathrm{R}$ & $\mathrm{R}$ & $\mathrm{R}$ & $\mathrm{R}$ & $\mathrm{R}$ & $\mathrm{R}$ & $\mathrm{R}$ & $\mathrm{R}$ & $\mathrm{R}$ & $\mathrm{R}$ & $\mathrm{R}$ & $\mathrm{R}$ & $\mathrm{R}$ & $\mathrm{R}$ & $\mathrm{R}$ & $\mathrm{R}$ & $\mathrm{R}$ & $\mathrm{R}$ & $\mathrm{R}$ & $S$ \\
\hline 35 & SB-35 & $S$ & $S$ & $S$ & $S$ & $S$ & $S$ & $S$ & $S$ & $S$ & S & $\mathrm{R}$ & $\mathrm{R}$ & $S$ & $S$ & $S$ & $S$ & $S$ & $S$ & $S$ & $S$ \\
\hline 36 & SB-36 & $S$ & $S$ & $S$ & $S$ & $S$ & $S$ & $S$ & $S$ & $S$ & $S$ & $S$ & $S$ & $S$ & $S$ & $S$ & $S$ & $S$ & $S$ & $S$ & $S$ \\
\hline 37 & SB-37 & $S$ & $S$ & $S$ & $S$ & $S$ & $S$ & $\mathrm{R}$ & $S$ & $S$ & S & $\mathrm{R}$ & $S$ & $S$ & $S$ & $S$ & $S$ & $S$ & $S$ & $\mathrm{R}$ & $\mathrm{R}$ \\
\hline 38 & SB-38 & $S$ & $S$ & $S$ & $S$ & $S$ & $S$ & $S$ & $S$ & $S$ & S & S & $\mathrm{R}$ & $S$ & $S$ & $\mathrm{R}$ & $S$ & $S$ & $S$ & $S$ & $S$ \\
\hline 39 & SB-39 & $S$ & $S$ & $S$ & $S$ & $S$ & $S$ & $S$ & $S$ & $S$ & $S$ & $S$ & $S$ & $S$ & $S$ & $S$ & $S$ & $S$ & $S$ & $S$ & $S$ \\
\hline 40 & SB-40 & $S$ & $S$ & $S$ & $S$ & $S$ & $S$ & $\mathrm{R}$ & $S$ & S & S & $\mathrm{R}$ & $S$ & $S$ & $S$ & $\mathrm{R}$ & $S$ & $S$ & $S$ & $S$ & $\mathrm{R}$ \\
\hline 41 & SB-41 & $S$ & $\mathrm{R}$ & $S$ & $S$ & $S$ & $S$ & $S$ & $S$ & $S$ & $S$ & $S$ & $S$ & $S$ & $S$ & $S$ & $S$ & $S$ & $S$ & $S$ & $S$ \\
\hline 42 & SB-42 & $S$ & $S$ & $S$ & $S$ & $S$ & $S$ & $S$ & $S$ & S & S & $\mathrm{R}$ & $\mathrm{R}$ & $S$ & $S$ & $S$ & $S$ & $S$ & $S$ & $S$ & $S$ \\
\hline 43 & SB-43 & $S$ & $S$ & $S$ & $S$ & $S$ & $S$ & $S$ & $S$ & $S$ & $S$ & S & $S$ & $S$ & $S$ & $\mathrm{R}$ & $\mathrm{S}$ & $S$ & $S$ & $S$ & $S$ \\
\hline 44 & SB-44 & $S$ & $S$ & $S$ & $S$ & $S$ & $S$ & $S$ & $S$ & $S$ & $S$ & S & $S$ & $S$ & $S$ & $S$ & $S$ & $S$ & $S$ & $S$ & $\mathrm{R}$ \\
\hline 45 & SB-45 & $S$ & $S$ & $S$ & $S$ & $S$ & $S$ & $S$ & $S$ & S & S & S & $S$ & $S$ & $S$ & $S$ & $S$ & $S$ & $S$ & $S$ & $S$ \\
\hline
\end{tabular}

R: resistant ; S: sensitive; AMX: amoxicillin; AMC: amoxicillin-clavulanic acid; ATZ: aztreonam; C: chloramphenicol; CTR: ceftriaxone; CPD: cefpodoxime; CAZ co-trimoxazole; ERP: ertapenem; F/M: nitrofurantoin; FOX: cefoxitin; GEN : gentamicin; IMP: imipenem; K-kanamycin, NX: norfloxacin; MRP- meropenem; TE: $t$

Citations are available as wild birds as reservoirs of extended-spectrum $\beta$-lactamase (ESBL) and AmpC $\beta$-lactamase producing E. coli (Alcala et al. 2016). Interestingly, a significant percentage of MDR E. coli isolates detected in black-headed gulls (3.3\%), starlings (19.2\%), herring gulls (3.3\%), deer (3.3\%) of Ireland (Carroll et al. 2015). Screening for carbapenem resistance showed that $11.11 \%(5 / 45)$ isolates were resistant to any carbapenem drugs. Three carbapenem-resistant isolates showed keyhole between EDTA and carbapenem drugs, indicating metallo beta-lactamase production (MBL). A conservative approach was utilized for genotypic screening, and only isolated classified as resistant were analysed for AMR encoding genes. All the ESBL producing isolates were MDR type and genotypic screening of the ESBL positive isolates $(n=17)$ revealed blaCTX-M-1 $(n=10)$, blaCTX-M-15 ( $n=7)$ and other antibiotic resistance genes like blaTEM $(n=6)$, blaAmpC $(n=4)$, qnrS $(n=6)$, qnrA $(n=3)$, qnrB $(n=3)$, sull $(n=7)$, sulll $(n=3)$, tetA $(n=4)$, tetB $(n=5)$. Similarly, most of the ESBL E. coli isolates in wild birds were multi-drug resistant, and the most common resistant phenotype are beta-lactams, quinolones, tetracycline, and sulfamethoxazole/trimethoprim (Alcala et al. 2016). The dominant extended-spectrum cephalosporin resistance genes in animals were blaCTX-M-1, while in humans were blaCTX-M-15 and blaCTX-M-14. However, recent studies showed that the prevalence of CTX-M-1 genes in humans (Madec et al. 2015) and rare identification of CTX-M-15 in animal isolates (Dahmen et al. 2013). In the present study, E. coli isolated from sloth bear harbored both CTX-M-1 and CTX-M-15 variants indicated sharing of gene pool. Screens for carbapenemase genes revealed three isolates were positive for the blaNDM-5 gene. All five carbapenem resistant isolates were MDR ESBL producers. The bla NDM positive isolates also co-harboured blaCTX-M1/15, blaTEM-1, qnrS, qnrA, qnrB, su/1, tetA and tetB genes.

The efflux pump-mediated carbapenem resistance was noticed in two $(40 \%, 2 / 5)$ isolates. Efflux pump mediated carbapenem resistance was noticed in $E$. coli isolated from piglets and dairy calves (Pruthvishree et al. 2017; Murugan et al. 2019). Chloramphenicol (catl, floR), aminoglycoside (ac (3)-IV, aph (3')-Ila), quinolone (qnrS, qnrA, qnrB), trimethoprim (dhfrl), sulphadimidine (sull, sullI) and tetracycline resistance genes (tetA, tetB) were commonly detected in the phenotypic resistance isolates. Furness et al. (2017) reported $E$. coli from the faeces of small free-living mammals were resistant to trimethoprim, ampicillin, ciprofloxacin, and cefotaxime. Screens for virulence genes revealed that five ESBL producing and one carbapenem-resistant isolates harbored any of the virulence genes screened (Table 1). Reports were available in India on ESBL producing and carbapenem resistant $E$. coli harboring virulence genes in animals (Pruthvishree et al. 2017; Nirupama et al. 2018; Pruthvishree et al. 2018). Plasmid replicon typing of the three blaNDM-5gene-positive E. coli revealed that the NDM gene was on Incl1 plasmid in two isolates (SB27/ 17-CRE, SB28/ 12-CRE) and IncF plasmid was on one isolate (SB28/ 16-CRE). The Incl1 and IncF plasmids also co-harbored PMQR, extended-spectrum cephalosporin, sulphonamide, and tetracycline resistance genes. The IncF plasmids have been reported worldwide among Enterobacteriaceae and are one of the most prevalent incompatibility types involved in the transfer of resistance determinants (Yang et al. 
2015). Recently, IncF plasmid carrying multi-drug resistant genes were reported in Shigella flexneri isolates from India (Sethuvel et al. 2019). In our study, the Incl1 and IncF plasmids also co-harbored blaCTX-M-15, blaTEM-1, tetA, and sull AMR genes. Similarly, IncF plasmid encoding beta-lactamase (NDM-1, OXA-1), aminoglycoside (aacA4, aadA2, and aacC2), and extended-spectrum cephalosporin resistance (CTX-M-15) genes from E.coli ST131 was reported (Bonnin et al. 2012). The plasmid multilocus sequence typing (pMLST) of the blaNDM isolates showed ST 297. Recently we reported blaVIM positive E.coli to isolate with ST 297 (Murugan et al. 2019).

The ESBL producing, carbapenem and multidrug resistant E. coli were divided into three groups in the principal component analysis (PCA). The first two dimensions which included showed the most variance of data (first: $62.5 \%$; second: $7.3 \%$ ). The third and fourth dimensions, despite being significant, were not plotted due to the complexity of the corresponding output (Fig. 1). Similar PCA analysis of ESBL producing E. coli isolates were carried out in piglets and pig farm workers (Tamta et al. 2020).

In conclusion, the study documented the presence of multi-drug resistant (MDR), ESBL producing, and carbapenem resistant $E$. coil carrying virulence genes. The isolates co-harboring antibiotic resistant with virulence genes are critically important in public health point of view. To the best of our knowledge, this is the first report of IncF and Incl1 type plasmids carrying multidrug-resistant and NDM carbapenamse producing genes in sloth bear.

\section{Declarations}

\section{Acknowledgements}

The authors would like to acknowledge the financial assistance provided by National Referral Centre on Wildlife Healthcare (CZA, New Delhi) for supporting the research work. The authors would further like to thank Director, IVRI and Wildlife SOS for permitting to carry out the research work.

\section{Ethical Approval}

Not applicable

\section{Consent to Participate}

Not applicable

\section{Consent to Publish}

We give consent to publish

\section{Authors Contributions}

Vinodh Kumar O. R - Conceptualization, Data curation, Formal analysis, Writing - original draft

M.Karikalan - Data curation, Formal analysis, Writing - original draft.

S. Ilayaraja - Methodology, Data curation

B.R Singh - Validation

Arun A Sha- Methodology, Resources, Software

D.K.Sinha- Validation

S. Chandra Mohan - review \& editing

A.M.Pawde - review \& editing

AK Sharma- Validation

\section{Funding}

This research did not receive any specific grant from funding agencies in the public, commercial, or not-for-profit sectors

\section{Competing Interests}

The authors declare no conflict of interest. 


\section{Availability of data and materials}

All data generated or analysed during this study are included in this published article

\section{References}

1. Alcala L, Alonso CA, Simon C, González-Esteban G, Orós J, Rezusta A, Torres C (2016) Wild birds, frequent carriers of extended-spectrum $\beta$-lactamase (ESBL) producing E. coli of CTX-M and SHV-12 types. Microbial ecology 72:861-869

2. Allen HK, Donato J, Wang HH, Cloud-Hansen KA, Davies J, Handelsman J (2010) Call of the wild: antibiotic resistance genes in natural environments. Nature Reviews Microbiology 8:251

3. Alroy K, Ellis JC (2011) Pilot study of antimicrobial-resistant Escherichia coli in herring gulls (Larus argentatus) and wastewater in the northeastern United States. Journal of Zoo and Wildlife Medicine 42:160-163

4. Andrews J (2003) Detection of extended-spectrum b-lactamases (ESBLs) in E. coli and Klebsiella species. British Society for Antimicrobial Chemotherapy

5. Atterby C, Börjesson S, Ny S, Järhult JD, Byfors S, Bonnedahl J (2017) ESBL producing E. coli in Swedish gulls - A case of environmental pollution from humans? PloS one 12:e0190380

6. Bonnedahl J, Drobni M, Gauthier-Clerc M, Hernandez J, Granholm S, Kayser Y, Olsen B (2009) Dissemination of Escherichia coli with CTX-M type ESBL between humans and yellow-legged gulls in the south of France. PloS one 4:e5958

7. Bonnin RA, Poirel L, Carattoli A, Nordmann P (2012) Characterization of an IncFll plasmid encoding NDM-1 from E. coli ST131. PLoS One 7:e34752

8. Carroll D, Wang J, Fanning S, McMahon BJ (2015) Antimicrobial resistance in wildlife: implications for public health, Zoonoses and public health. 62:534542

9. CLSI (2014) Performance standards for antimicrobial susceptibility testing, Clinical and Laboratory Standard Institute. CLSI document, Wayne, PA M100S124

10. Dahmen S, Haenni M, Chatre P, Madec JY (2013) Characterization of blaCTX-M IncFIl plasmids and clones of E. coli from pets in France. Journal of Antimicrobial Chemotherapy 68:2797-2801

11. Dolejska M, Cizek A, Literak I (2007) High prevalence of antimicrobial-resistant genes and integrons in E. coli isolates from black-headed gulls in the Czech Republic. Journal of Applied Microbiology 103:11-19.

12. Furness LE, Campbell A, Zhang L, Gaze W, McDonald RA (2017) Wild small mammals as sentinels for the environmental transmission of antimicrobial resistance. Environmental research 154:28-34

13. Johnson TJ, Wannemuehler YM, Johnson SJ, Logue CM, White DG, Doetkott C, Nolan LK (2007) Plasmid replicon typing of commensal and pathogenic Escherichia coli isolates. Applied and Environmental Microbiology 73:1976-1983

14. Kummerer K (2009) Antibiotics in the aquatic environment-a review-part I. Chemosphere 75:417-434

15. Madec JY, Haenni M, Métayer V, Saras E, Nicolas-Chanoine MH (2015) High prevalence of the animal-associated blaCTX-M-1 Incl1/ST3 plasmid in human E. coli isolates. Antimicrobial agents and chemotherapy 59:5860-5861

16. Murugan MS, Sinha DK, VinodhKumar OR, Yadav AK, Pruthvishree BS, Vadhana P, Nirupama KR, Baradwaj M, Singh BR (2019) Epidemiology of carbapenem-resistant Escherichia coli and first report of blaVIM carbapenemases gene in calves from India. Epidemiology and infection (Accepted)

17. Nirupama KR, Vinodh Kumar OR, Pruthvishree BS, Sinha DK, Murugan MS, Krishnaswamy N, Singh BR (2018) Molecular characterization of blaOXA-48 carbapenemase, extended-spectrum $\beta$-lactamase-and Shiga toxin-producing $E$. coli isolated from farm piglets in India. Journal of global antimicrobial resistance 13:201-205

18. Pruthvishree BS, Vinodh Kumar OR, Sinha DK, Malik YS, Dubal ZB, Desingu PA, Singh BR (2017) Spatial molecular epidemiology of carbapenem-resistant and New Delhi metallo beta-lactamase (blaNDM) producing E. coli in the piglets of organized farms in India. Journal of applied microbiology 122:15371546

19. Pruthvishree BS, Vinodh Kumar OR, Sivakumar M, Tamta D, Sunitha R, Singh BR (2018) Molecular characterization of extensively drug resistant (XDR), extended spectrum beta-lactamases (ESBL) and New Delhi Metallo beta-lactamase-1 (blaNDM1) producing E. coli isolated from a male dog. Veterinarski arhiv 88:139-148

20. Sethuvel DPM, Anandan S, Ragupathi NKD, Gajendiran R, Kuroda M, Shibayama K, Veeraraghavan B (2019) IncFIl plasmid carrying antimicrobial resistance genes in Shigellaflexneri: Vehicle for dissemination. Journal of global antimicrobial resistance 16:215-219

21. Tamta S, VinodhKumar OR, Pruthvishree BS, Karthikeyan R, Rupner RN, Chethan GE, Dubal ZB, Sinha DK, Singh BR (2020) Faecal carriage of extended spectrum beta-lactamase (ESBL) and New Delhi metallo beta-lactamase (NDM) producing Escherichia coli between piglets and pig farmworkers. Comparative Immunology, Microbiology and Infectious Diseases 73:101564

22. Yang QE, Sun J, Li L, Deng H, Liu BT, Fang LX, Liu YH (2015) IncF plasmid diversity in multi-drug resistant E. coli strains from animals in China. Frontiers in microbiology 6:964

\section{Figures}




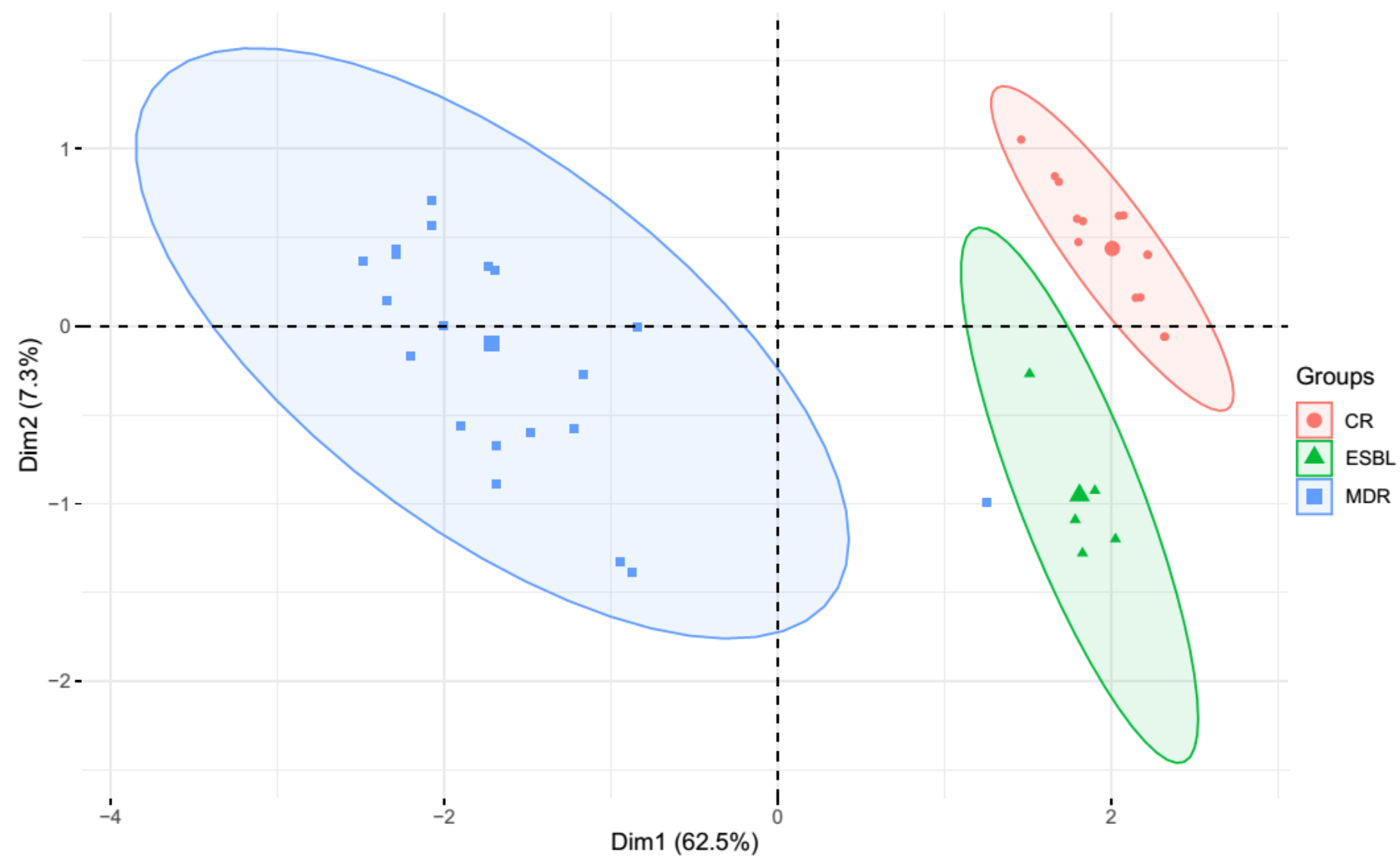

Figure 1

Principal Component Analysis (PCA) plots of the drug resistant E. coli isolates. The E. coli are encompassed in $95 \%$ confidence intervals grouping based on the resistant group [ Carbapenem (CR), Extended Beta-lactam (ESBL), Multidrug resistant (MDR) ]. 